\title{
ORIGINAL ARTICLE \\ Pharmacokinetics of the ghrelin agonist capromorelin in a single ascending dose Phase-I safety trial in spinal cord-injured and able-bodied volunteers
}

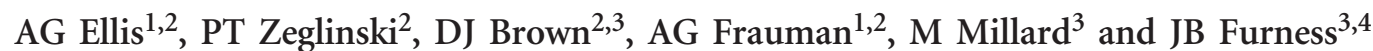

Study design: Single centre, single ascending dose study.

Objectives: To compare the pharmacokinetics and assess the safety of capromorelin, a compound that has potential to treat constipation following spinal cord injury (SCI), in groups of able-bodied and $\mathrm{SCl}$ volunteers.

Setting: Local population from Victoria, Australia.

Methods: Following initial screening and baseline blood collections, participants received ascending oral doses (20, 50 and then $100 \mathrm{mg}$ at least 1-week apart) of capromorelin after pre-dose blood collection, followed by blood collections over the following $12 \mathrm{~h}$ for pharmacokinetic analysis and 1-week and 4-week follow-up blood collections for safety evaluations. Blood pressure and heart rate were monitored.

Results: No serious adverse events were recorded following any dose in either the able-bodied group or the $\mathrm{SCl}$ group. There were no abnormal blood pressure or heart rate changes. Minor adverse events resolved quickly without the need for treatment. Pharmacokinetic behaviour was broadly similar between groups, with both exhibiting dose-dependent increases in $C_{\max }$ and $\mathrm{AUC}_{0-\infty}$. The $\mathrm{SCl}$ participants showed greater variance in pharmacokinetic parameters and had a slightly delayed $T_{\max }$ and half-life.

Conclusion: Capromorelin at the doses tested was safe and well tolerated in both SCl and able-bodied participants and also showed similar pharmacokinetics with dose-dependent increases in concentration and drug exposure.

Sponsorship: Support for the study was provided by the Victorian State Government Transport Accident Commission.

Spinal Cord (2015) 53, 103-108; doi:10.1038/sc.2014.218; published online 2 December 2014

\section{INTRODUCTION}

An inability to empty the bowel at a convenient time or to prevent leakage events at inappropriate times are major issues identified in spinal cord injury (SCI). In many patients this is the most distressing aspect of SCI. ${ }^{1}$ Disturbances of bowel function occur in over $80 \%$ of people with SCI. ${ }^{2}$

Treatment of the inability to evacuate the bowel in patients with SCI generally involves oral laxatives, manual evacuation, anal dilatation, suppositories or enemas. ${ }^{1}$ There have been only a small number of investigations of prokinetic drugs, neostigmine ${ }^{3,4}$ and prucalopride. ${ }^{5}$ Neostigmine is an anti-cholinesterase, chosen for its ability to enhance excitatory cholinergic neural transmission to the muscle of the colorectum, and to enhance normal neural control of defecation from pathways within the bowel wall. However, this drug affects cholinergic transmission at other sites, including enhancing cholinergic transmission to the cardiovascular system, respiratory system and skeletal muscle. The investigators used the muscarinic antagonist, glycopyrrolate, to reduce some of the side effects. ${ }^{4}$ Prucalapride is a 5HT4 receptor agonist that acts on enteric neurons to augment bowel contractions. An investigation of prucalapride showed that the compound was effective in decreasing bowel transit times and increasing the frequency of bowel movements, but it has side effects, notably in causing headache, and about half the participants withdrew. $^{5}$

Another class of prokinetic drugs has been discovered recently. These are centrally penetrant ghrelin receptor agonists that stimulate neurons in the spinal defecation centres. ${ }^{6,7}$ As the majority of spinal cord injuries are at levels rostral to the defecation centres at L5-S4, these compounds have the potential to be used to treat constipation in SCI and thus decrease the chances of incontinence.

In an animal model of SCI, the ghrelin receptor agonist capromorelin caused defecation after SCI with a similar potency as in normal animals. ${ }^{8}$

Capromorelin has been in a limited number of human trials previously-for example, as a growth hormone secretogogue in the elderly ${ }^{9,10}$ or in treating gastro-oesophageal reflux. ${ }^{11}$ However, it has never been administered in the SCI setting. Given that there is a possibility of different pharmacokinetic behaviour in SCI compared with able-bodied people ${ }^{12}$ an evaluation of the pharmacokinetics, safety and tolerability of capromorelin was undertaken.

The doses selected for the study were based on the doses previously used in clinical studies and consideration of the no-observed-adverse-

${ }^{1}$ Clinical Pharmacology and Therapeutics, Austin Health, Heidelberg, Victoria, Australia; ${ }^{2}$ Department of Medicine, University of Melbourne, Austin Health, Heidelberg, Victoria, Australia; ${ }^{3}$ Spinal Research Institute, Royal Talbot Rehabilitation Centre, Victoria, Australia and ${ }^{4}$ Department of Anatomy and Neuroscience, University of Melbourne, Parkville, Victoria, Australia

Correspondence: AG Ellis, Clinical Pharmacology and Therapeutics, Department of Medicine, University of Melbourne, Austin Health Level 5 Lance Townsend Building, 145 Studley Road, Heidelberg, Victoria 3084, Australia.

E-mail: andrew.ellis@austin.org.au

Received 24 July 2014; accepted 30 October 2014; published online 2 December 2014 
effect-levels in animals. A dose of $20 \mathrm{mg}$ was previously used for a multi-dose study for treating gastro-oesophageal reflux disease, but the effects did not reach clinical significance. ${ }^{11}$ Dose levels were based on $20 \mathrm{mg}$ being at threshold for clinical effectiveness and $100 \mathrm{mg}$ being less than $20 \%$ of animal no-observed-adverse-effect-levels and $1 / 3$ of the highest doses previously used safely in humans.

The purpose of this study was to evaluate the safety profile, tolerability and pharmacokinetics following single oral doses of 20, 50 and $100 \mathrm{mg}$ of capromorelin in able-bodied participants and in SCI participants.

\section{MATERIALS AND METHODS}

The study was approved by the Austin Health Human Research Ethics Committee and performed at the Austin Centre for Clinical studies ('Nucleus Network'). All applicable institutional and governmental regulations concerning the ethical use of human volunteers were followed during the course of this research.

\section{Participant eligibility}

All participants underwent initial screening and were fully informed, and signed protocol-specific informed consent was obtained. Any participant was free to withdraw from the study at any time for any reason. Candidates were excluded if they were $<18$ years or $>45$ years old, were females of child-bearing potential or pregnant or breastfeeding, were unhealthy (as defined by significant deviation from normal medical history or aberrant results from physical examination/electrocardiogram/clinical laboratory determinations), or had a history of toxicities or allergy related to previous treatments. Spinal cordinjured candidates from the Victorian Spinal Cord Service at Austin Health with spinal injuries between T6 and T12 and not otherwise excluded as per exclusion criteria applied to able-bodied candidates described above were invited to participate

Any candidates were excluded if they were receiving drugs known to inhibit CYP3A4 (indinavir, nelfinavir, ritonavir, clarithromycin, itraconazole, ketoconazole, nefazodone, saquinavir, telithromycin, aprepitant, erythromycin, fluconazole, verapamil, diltiazem and cimetidine) or induce CYP3A4 (efavirenz, nevirapine, barbiturates, carbamazepine, glucocorticoids, modafinil, oxacarbazepine, phenytoin, pioglitazone, rifabutin, rifampin and St John's wort). The consumption of grapefruit or cranberry juice was also banned from 5 days prior to the study and during the study period.

\section{Safety/tolerability}

Physical examinations were performed at pre-study screening and on the day of each dose plus follow-up at +1 week and +4 weeks following the final dose, and included body measurements, vital signs, electrocardiogram, blood and urine tests and urine drug screening and pregnancy test. Adverse events and any concomitant medications were monitored throughout the study. Defecation was recorded but was not a target measure in this safety study. Blood analysis included urea, haematocrit, bilirubin, total $\mathrm{CO} 2$, red cell count, white cell count, creatinine, sodium, potassium, chloride, monocytes, platelets, lymphocytes, neutrophils, basophils, alkaline phosphatase, albumin, total protein, mean corpuscular volume, eosinophils, gamma glutamyl transpeptidase, alanine transaminase, mean corpuscular haemoglobin, globulin and haemoglobin.

Urine analysis included $\mathrm{pH}$, glucose, bilibruin, ketone, specific gravity, blood, protein, urobilinogen, nitrite and leukocytes.

\section{Procedure}

Participants received the lowest dose of capromorelin $(20 \mathrm{mg})$ initially. Subsequent higher doses $(50$ and $100 \mathrm{mg}$ ) were received after a minimum 1 week washout period between doses and only if safety and tolerability assessments were acceptable. Doses of capromorelin tartrate were prepared as capsule formulation and administered orally with $240 \mathrm{ml}$ water following a $12 \mathrm{~h}$ fasting period.

\section{Pharmacokinetics}

Blood sample $(5 \mathrm{ml})$ collections for capromorelin analysis were taken from an indwelling catheter in the cubital vein at $-30 \mathrm{~min}$ (pre-dose) and at $+20 \mathrm{~min}$, $+30 \mathrm{~min},+40 \mathrm{~min},+1 \mathrm{~h},+1.5 \mathrm{~h},+2 \mathrm{~h},+2.5 \mathrm{~h},+3 \mathrm{~h},+3.5 \mathrm{~h},+4 \mathrm{~h},+5 \mathrm{~h},+6 \mathrm{~h}$, $+7 \mathrm{~h},+8 \mathrm{~h}$ and $+12 \mathrm{~h}$. Red cells were separated and plasma aliquoted prior to freezing at $-20^{\circ} \mathrm{C}$ and transfer within $24 \mathrm{~h}$ to be stored at $-70^{\circ} \mathrm{C}$ until analysis. Capromorelin levels were measured at Austin Health, Melbourne, VIC, Australia, using a liquid chromatography-tandem mass spectrometry (LCMS/MS) validated assay. Briefly, $500 \mu \mathrm{l}$ of plasma was extracted using 50:50, hexane:ethyl acetate, $\mathrm{v} / \mathrm{v}$, after the addition of an internal standard (stable $\mathrm{d}_{7}$-labelled capromorelin, synthesised at Walter and Eliza Hall Institute, Melbourne, VIC, Australia). Supernatants were dried under a stream of air and then reconstituted in the starting mobile phase before being injected onto the LC-MS/MS system (6460 QQQ Agilent Technologies, Melbourne, VIC, Australia) and chromatographed using an acetonitrile gradient elution from a Zorbax-C18 rapid resolution HT $50 \times 2.1 \mathrm{~mm}$ column (Agilent Technologies), and electrospray ionisation in positive ion mode delivered analytes to the tandem mass spectrometry detector. Capromorelin concentration was interpolated from a multipoint standard curve ranging from $20 \mathrm{pg} \mathrm{ml}^{-1}$ to 100 $\mu \mathrm{g} \mathrm{ml}^{-1}$. Validation of the method (accuracy, precision, recovery, stability and interference) followed laboratory standard operating procedures.

Noncompartmental pharmacokinetic analyses were performed using PK Solutions, version 2.0 (Summit Research Services, Montrose, CO, USA), and Excel 2007 (Microsoft, Sydney, NSW, Australia).

Capromorelin tartrate was supplied by RaQualia Pharma Incorporated, Nagoya, Japan, who also supplied confidential in-house safety and drug metabolism data.

\section{RESULTS}

\section{Participation}

Participant characteristics are summarised in Table 1. All participants were male. Six SCI participants were recruited for the study and one SCI participant withdrew after the first dose for personal reasons (relocating).

\section{Safety/tolerability}

No serious adverse events were recorded following any dose of capromorelin in either the able-bodied group $(n=10)$ or the SCI group $(n=6)$. Minor adverse events relating to drug administration included increased perspiration (7) or warm sensation (2), abdominal discomfort or spasm (3), lethargy/drowsiness/lightheadedness (5), palpitations (1) or lower back pain (1); all were resolved within $1-8.5 \mathrm{~h}$ without the need for treatment or other actions. The incidence of minor adverse events per participant was $\sim 2.5$ times higher in the able-bodied group (1.7) compared with the SCI group (0.7). Recorded defecation events commenced at approximately 60-90 min. No significant trends in defecation volume, rate or consistency of stools were observed between the different doses in either group (data not presented).

No significant changes were noted in the results for physical examinations, electrocardiograms, vital signs, urinalysis or laboratory

\section{Table 1 Characteristics of participants}

\begin{tabular}{|c|c|c|c|}
\hline Characteristic & Able-bodied & $S C l$ & P-value \\
\hline$N$ & 10 & 6 & \\
\hline Age (years) & $25.4 \pm 3.9$ & $27.5 \pm 3.8$ & 0.31 \\
\hline Gender (male:female) & 10:0 & $6: 0$ & - \\
\hline Height $(\mathrm{cm})$ & $180.9 \pm 7.8$ & $181.0 \pm 3.2$ & 0.98 \\
\hline Weight (kg) & $76.8 \pm 11.3$ & $90.5 \pm 14.8$ & 0.05 \\
\hline BMI $\left(\mathrm{kg} \mathrm{m}^{-2}\right)$ & $23.7 \pm 3.3$ & $27.7 \pm 4.5$ & 0.07 \\
\hline
\end{tabular}

Abbreviations: BMI, body mass index; $\mathrm{SCl}$, spinal cord injury. Data are mean \pm s.d. The $P$-values refer to differences across groups using analysis of variance. 
tests on blood samples (full blood examination, urea and electrolytes and liver function tests; see Methods) taken before or after dosing and at follow-up time points, with the exception of the urine $\mathrm{pH}$ of one
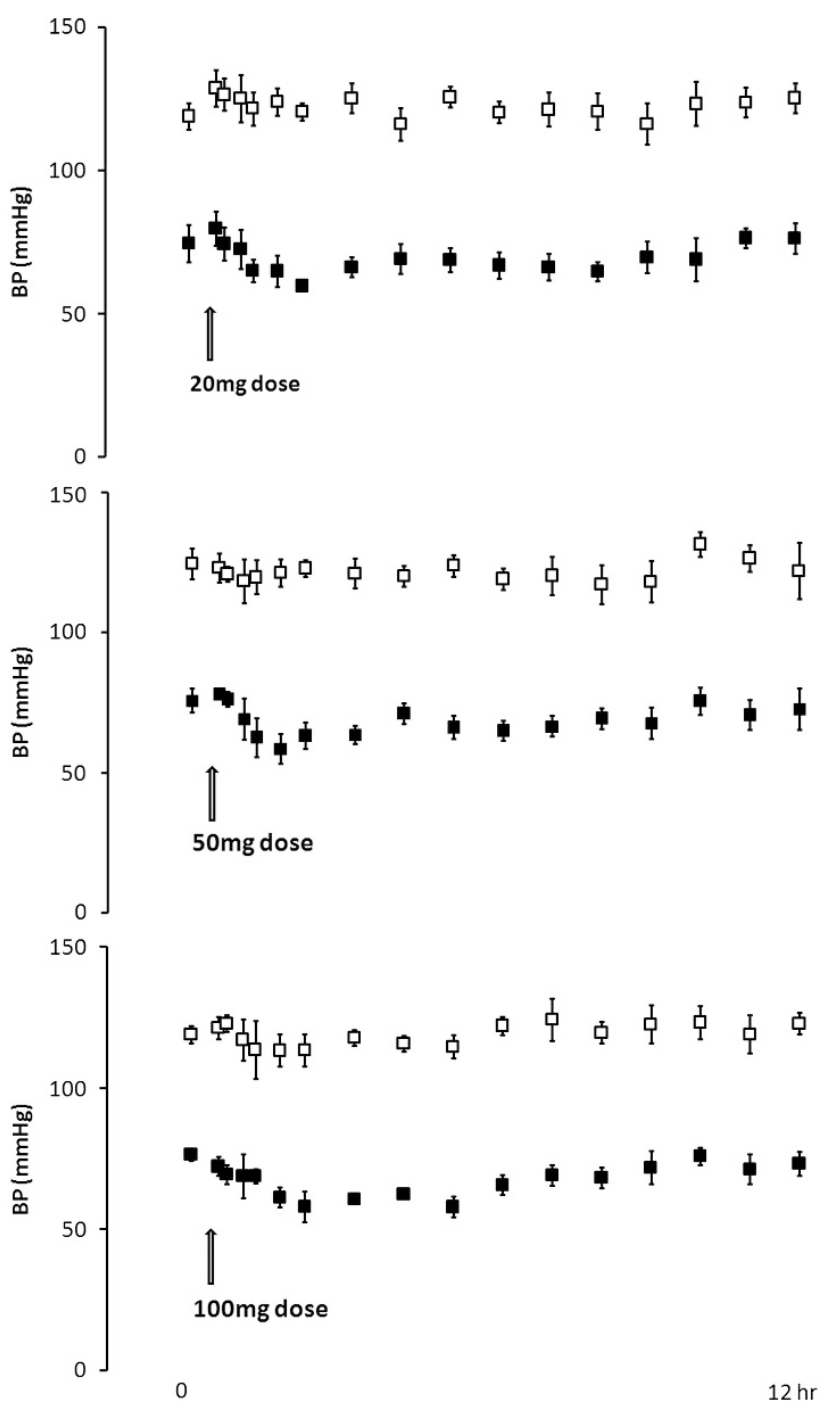

Figure 1 Blood pressure data in $\mathrm{SCl}$ participants at each dose level for the period from pre-dose to $+12 \mathrm{~h}$ post dose (open squares systolic, filled squares diastolic, Mean \pm s.e.m., $n=4-6)$.
SCI participant that showed $\mathrm{pH} \geqslant 9.0$ for both pre-study and during study samples. Greater variability was generally noted in the SCI group than in the able-bodied group.

No significant alteration in heart rate, respiration rate or blood pressure was evident in either group of participants. Of note, no effects on blood pressure for the SCI group during the period from pre-dose to $12 \mathrm{~h}$ post dose were seen (Figure 1).

\section{Pharmacokinetics}

The calculated non-compartmental pharmacokinetic parameters including half-life $\left(t_{1 / 2}\right)$, area under the curve from zero to infinity $\left(\mathrm{AUC}_{0-\infty}\right)$, maximum concentration $\left(C_{\max }\right)$ and time to $C_{\max }\left(T_{\max }\right)$ are presented in Table 2 along with linear (Figure 2) and semi-log (Figure 3) plots of plasma elimination of capromorelin. The pharmacokinetic profiles (Figures 2 and 3) and calculated parameters (Table 2) showed greater variability for the SCI group than for the able-bodied group at each dose level. However, a linear increase in $C_{\max }$ and $\mathrm{AUC}_{0-\infty}$ was evident with ascending dose (Figure 4). Doserelated increases were not evident with either volume of distribution or $T_{\max }$ (Figure 4 ).

Overall, pharmacokinetic parameters and peak plasma concentrations were broadly similar between the two groups (Table 2). There was slower elimination in the SCI group with significantly $(P<0.05)$ higher $t_{1 / 2}(3.10 \pm 0.53 \mathrm{~h})$ and a later $T_{\max }(1.70 \pm 0.45 \mathrm{~h})$ in the SCI group compared with able-bodied controls $\left(t_{1 / 2} 2.54 \pm 0.42 \mathrm{~h}\right.$ and $T_{\max }$ $0.88 \pm 0.31 \mathrm{~h}$ ) at the $50 \mathrm{mg}$ dose level. However, these differences were not significant at the 20 or $100 \mathrm{mg}$ dose levels.

\section{DISCUSSION}

Oral capromorelin was well tolerated in both SCI and able-bodied participants at the doses tested. No serious adverse events were encountered and no major changes in measured physiological parameters, either before dose, immediately following any dose or at follow-up at 1 and 4 weeks after the final dose. No subjects withdrew because of adverse effects, in contrast to a previous study using the prokinetic prucalopride in participants with SCI. ${ }^{5}$ These results are consistent with the safety profile of oral capromorelin reported in previous human studies using either single $20 \mathrm{mg}$ dose ${ }^{11}$ or multi-dose (10 mg twice daily for 12 months) regimens. ${ }^{9}$ Of interest was the absence of any effect on participants' blood pressure as had been reported in preclinical animal studies. ${ }^{6}$ This may reflect differences in blood pressure control between species, lower relative doses used in the human study or differences in route of administration

Table 2 Model-independent pharmacokinetic parameters in able-bodied and SCI participants, mean $( \pm$ s.d.)

\begin{tabular}{|c|c|c|c|c|c|c|}
\hline Dose (mg) & $\mathrm{n}$ & $C_{\max }\left(n g m l^{-1}\right)$ & $\mathrm{t} t / 2(h)$ & $A \cup C_{0-\infty}\left(n g h m l^{-1}\right)$ & $\mathrm{V}_{d}(m l)$ & $\mathrm{T}_{\max }(h)$ \\
\hline \multicolumn{7}{|c|}{ Able-bodied participants } \\
\hline 20 & 10 & $28.1( \pm 4.5)$ & $2.75( \pm 0.62)$ & $97.9( \pm 17.9)$ & $837049( \pm 270242)$ & $1.18( \pm 0.45)$ \\
\hline 100 & 10 & $187.1( \pm 36.4)$ & $2.56( \pm 0.30)$ & $527.8( \pm 102.0)$ & $720322( \pm 149723)$ & $0.90( \pm 0.16)$ \\
\hline \multicolumn{7}{|c|}{ SCI participants } \\
\hline 100 & 5 & $206.5( \pm 72.5)$ & $2.91( \pm 0.30)$ & $798.9( \pm 562.3)$ & $681473( \pm 287521)$ & $1.33( \pm 0.72)$ \\
\hline
\end{tabular}

Abbreviations: $\mathrm{AUC}_{0-\infty}$, area under the curve from zero to infinity; $C_{\max }$, maximum concentration; SCl, spinal cord injury; $T_{\max }$, time to $C_{\max }, V_{\mathrm{d}}$, volume of distribution.

Data are mean \pm s.d. The $P$-values refer to differences across groups using analysis of variance.

a $P<0.05$ for analysis of variance of $\mathrm{SCl}$ vs able-bodied group at the same dose level. 

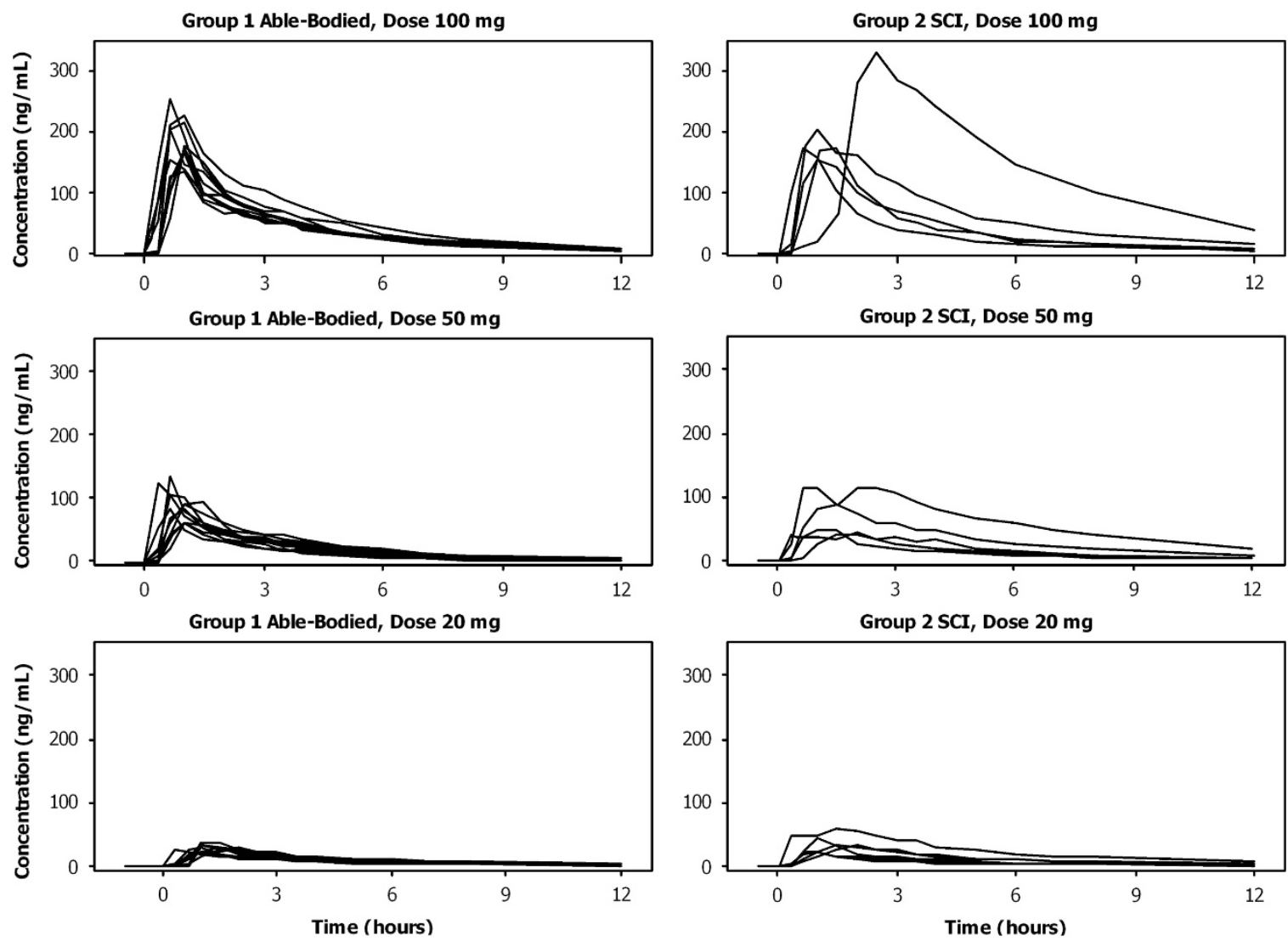

Figure 2 Individual plots of capromorelin elimination from plasma at 20, 50 and $100 \mathrm{mg}$ doses in able-bodied and SCl participants.
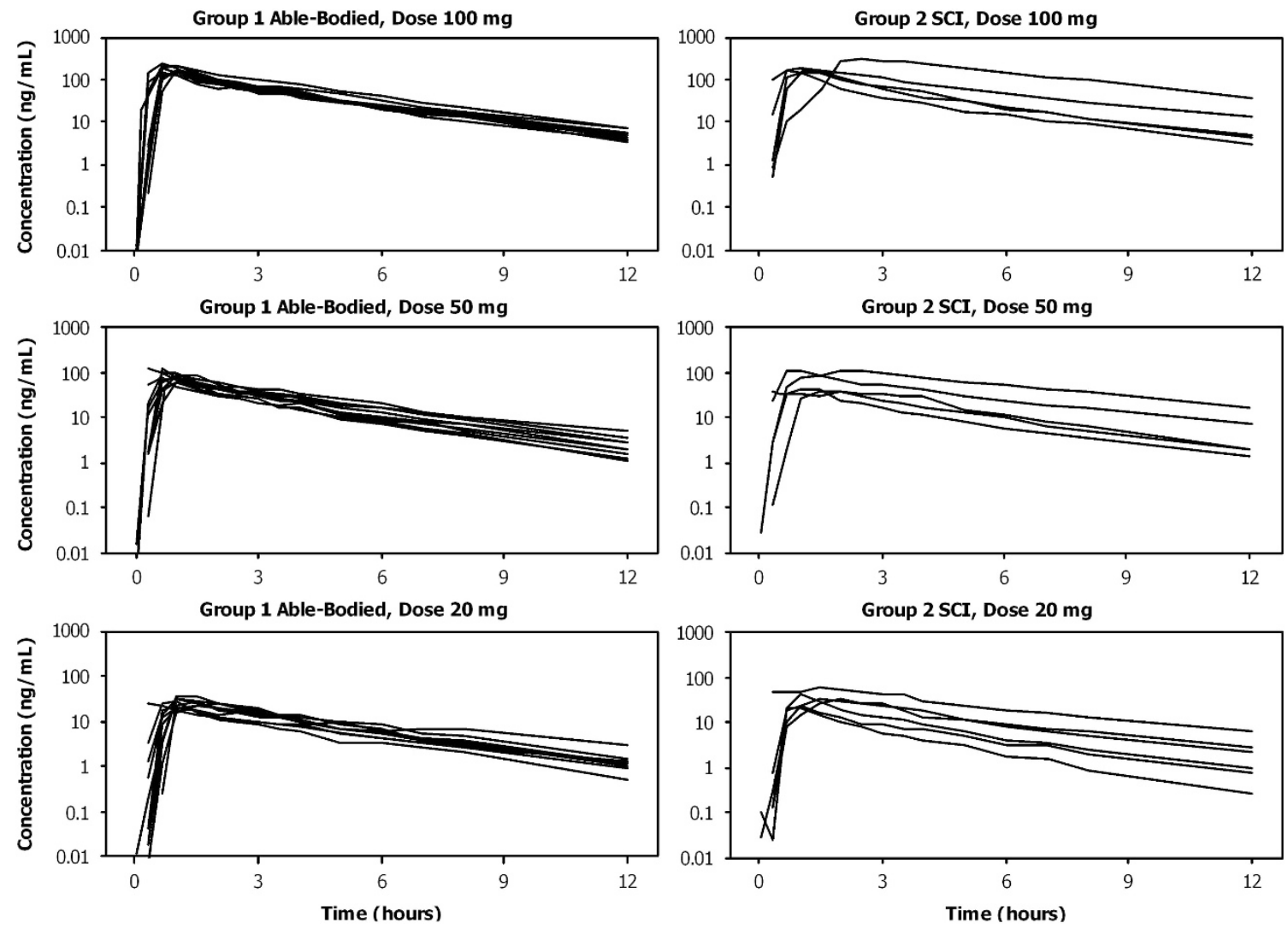

Figure 3 Individual semi-log plots of capromorelin elimination from plasma at 20, 50 and $100 \mathrm{mg}$ doses in able-bodied and SCl participants. 
a
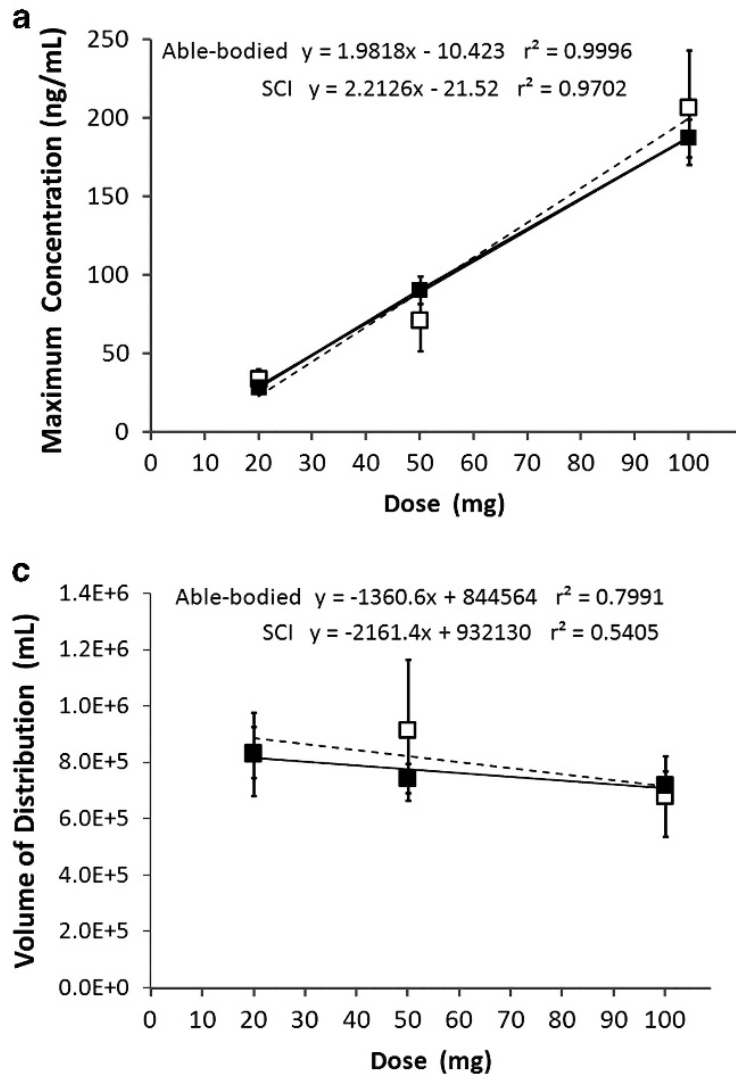

b

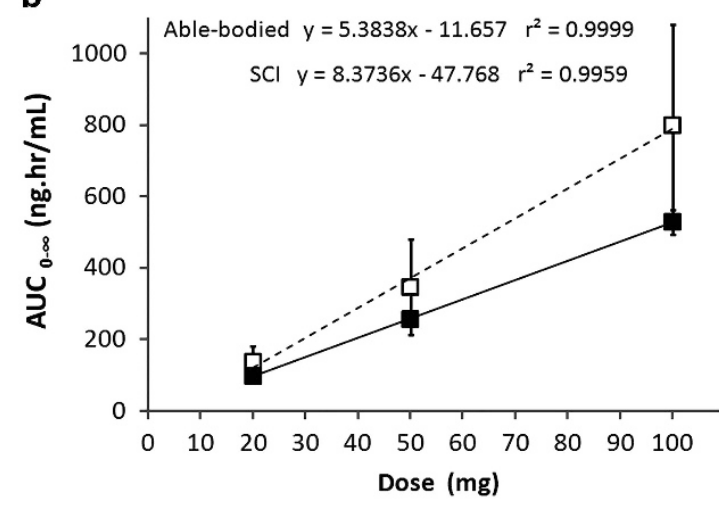

d

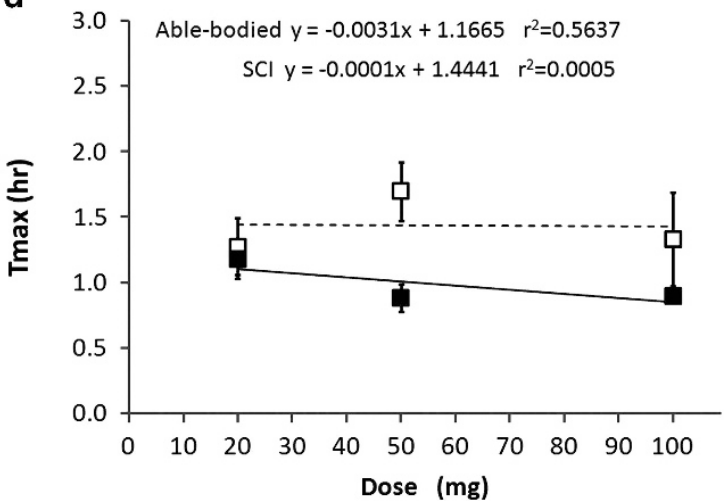

Figure 4 Plots of the relationship of major pharmacokinetic parameters including $C_{\max }$ (a), $A \cup C_{0-\infty}$ (b), $V_{d}$ (c) and $T_{\max }$ (d) with ascending dose of capromorelin. Mean \pm s.e.m. with linear regression fitted to the able-bodied group (filled squares and solid line) and the $\mathrm{SCl}$ group (open squares and dashed line).

(intravenous versus oral). There were no episodes of autonomic dysreflexia, consistent with the very low risk of occurrence seen in people with SCI injury below T6.

Participants in the SCI and the able-bodied groups were of the same gender and comparable age, height, weight and body mass index (Table 1). Greater variability in pharmacokinetic behaviour and slightly slower elimination of capromorelin in the SCI group versus the able-bodied group (Figures 2 and 3 ) are best explained by differences in rates of absorption and/or metabolism. Rates of absorption in SCI participants can potentially be affected by impaired postprandial gastric emptying. ${ }^{12}$ In addition, it is possible that enzymatic metabolism of capromorelin may have been affected by the permitted co-medications taken by SCI participants. A major pathway for metabolism of capromorelin is thought to be via enzymatic oxidation predominantly by CYP3A4 and CYP3A5. The SCI participants continued to receive their essential co-medications and the able-bodied participants took no co-medications during the study period. Therefore, potential remained for the medications taken by the SCI participants to alter the enzymatic metabolism of capromorelin. One SCI participant was noted to receive comedications that the other participants did not. Two of these medications were CYP3A substrates (amitriptyline and zolpidem) and enzyme interaction may explain this participant's Tmax and AUC that were, respectively, later and higher than that of any other participant at the same dose. Even with slightly more variance in the pharmacokinetic parameters for the SCI group it was evident that the pharmacokinetic behaviour in both groups was broadly similar, with both groups displaying dose-dependent increases in drug exposure $\left(\mathrm{AUC}_{0-\infty}\right)$ and concentration $\left(C_{\max }\right.$; Figures $4 \mathrm{a}$ and $\left.\mathrm{b}\right)$. The similarity between groups in pharmacokinetic behaviour with ascending oral doses up to $100 \mathrm{mg}$ and a lack of intolerance demonstrated in this study along with the previous safe administration of $10 \mathrm{mg}$ oral doses twice daily for a 12 -month period ${ }^{9}$ help to assure the safe performance in future studies that may trial this compound in SCI patients.

This was an open label study aimed at determining the safety, tolerability and pharmacokinetics of capromorelin. However, it was noted that bowel movements occurred at about $90 \mathrm{~min}$. The time to peak plasma concentration was $30-60 \mathrm{~min}$. This suggested that capromorelin might facilitate bowel emptying in people with SCI if taken about $1 \mathrm{~h}$ before their normal bowel emptying routine.

\section{DATA ARCHIVING}

There were no data to deposit.

\section{CONFLICT OF INTEREST}

The authors declare no conflict of interest.

\section{ACKNOWLEDGEMENTS}

The authors gratefully acknowledge the Victorian State Government Transport Accident Commission, through the Institute for Safety, Compensation and Recovery Research, for funding the study, all participants including relatives and caregivers of SCI participants for their cooperation, SCI research coordinator Janette Alexander for assistance in participant screening, Jonathon Baell from the Walter and Eliza Hall Institute Division of Medicinal Chemistry for synthesis of deuterated capromorelin and staff of the Nucleus Network Clinical Trials Unit, Austin Health. Dr Shinichi Koizumi of Raqualia Pharma 
Inc. kindly provided in-house data from previous trials of capromorelin in human subjects.

1 Coggrave MJ, Norton C. The need for manual evacuation and oral laxatives in the management of neurogenic bowel dysfunction after spinal cord injury: a randomized controlled trial of a stepwise protocol. Spinal Cord 2010; 48: 504-510.

2 Widerstrom-Noga EG, Felipe-Cuervo E, Broton JG, Duncan RC, Yezierski RP. Perceived difficulty in dealing with consequences of spinal cord injury. Arch Phys Med Rehabil 1999; 80: 580-586.

3 Korsten MA, Rosman AS, Ng A, Cavusoglu E, Spungen AM, Radulovic M et al. Infusion of neostigmine-glycopyrrolate for bowel evacuation in persons with spinal cord injury. Am J Gastroenterol 2005; 100: 1560-1565.

4 Rosman AS, Chaparala G, Monga A, Spungen AM, Bauman WA, Korsten MA. Intramuscular neostigmine and glycopyrrolate safely accelerated bowel evacuation in patients with spinal cord injury and defecatory disorders. Dig Dis Sci 2008; 53: 2710-2713.

5 Krogh K, Jensen MB, Gandrup P, Laurberg S, Nilsson J, Kerstens R et al. Efficacy and tolerability of prucalopride in patients with constipation due to spinal cord injury. Scand J Gastroenterol 2002; 37: 431-436.
6 Shimizu Y, Chang EC, Shafton AD, Ferens DM, Sanger GJ, Witherington J et al Evidence that stimulation of ghrelin receptors in the spinal cord initiates propulsive activity in the colon of the rat. J Physiol 2006; 576: 329-338.

7 Pustovit RV, Callaghan B, Kosari S, Rivera LR, Thomas H, Brock JA et al. The mechanism of enhanced defecation caused by the ghrelin receptor agonist, ulimorelin Neurogastroenterol Motil 2014; 26: 264-271.

8 Ferens DM, Habgood MD, Saunders NR, Tan YH, Brown DJ, Brock JA et al. Stimulation of defecation in spinal cord-injured rats by a centrally acting ghrelin receptor agonist. Spinal Cord 2011; 49: 1036-1041.

9 White HK, Petrie CD, Landschulz W, MacLean D, Taylor A, Lyles K et al. Effects of an oral growth hormone secretagogue in older adults. J Clin Endocrinol Metab 2009; 94 1198-1206.

10 Hersch EC, Merriam GR. Growth hormone (GH)-releasing hormone and GH secretagogues in normal aging: Fountain of Youth or Pool of Tantalus? Clin Interv Aging 2008; 3 $121-129$

11 Agrawal A, Gale JD, Sharma N, Blonski W, Hargreaves K, Allan R et al. Effect of single doses of capromorelin and ghrelin on esophageal reflux parameters and esophageal function: a randomized, double-blind, placebo-controlled study. Gasteroenterology 2009; 136: A120-A121.

12 Segal JL, Brunnemann SR. Clinical pharmacokinetics in patients with spinal cord injuries. Clin Pharmacokinet 1989; 17: 109-129. 